Weizmann Institute of Science in Rehovot, Israel, and his colleagues labelled proteins in human lung cancer cells with different fluorescent tags, and then tracked the proteins for two days after camptothecin treatment.

About one out of every 10,000 cells survived the treatment. The reason seems to lie in the behaviour of two of the labelled proteins, DDX5 and RFC1, levels of which increased in surviving cells but decreased in those that died.

\section{SYSTEMS BIOLOGY}

\section{Radical methods}

\section{Cell 135, 679-690 (2008)}

In one of the first demonstrations of the practical value of systems biology, James Collins and his colleagues at Boston University in Massachusetts have shown how the aminoglycoside class of antibiotics works. Although it was known that these compounds disrupt ribosomes - the cell's protein-making machinery - and cause proteins to be wrongly made, exactly how this killed bacteria was unclear.

Using DNA microarrays, Collins and his team mapped out changes in the geneexpression patterns of Escherichia coli treated with one or other of the aminoglycosides gentamicin and kanamycin. This allowed the researchers to pin down the gene networks that the drugs influenced. From these data and studies with bacterial mutants, they worked out that the movement of certain defective membrane proteins creates an electrochemical gradient across cell membranes that ultimately spawns harmful hydroxyl free radicals.

\section{MOLECULAR BIOLOGY}

\section{Cancer 101}

Science doi:10.1126/science.1165395 (2008) An RNA molecule called microRNA-101 inhibits the production and function of a protein that is found at high levels in many types of aggressive tumour.

Pinning this down first required Arul Chinnaiyan of the University of Michigan Medical School in Ann Arbor and his co-workers to predict the various microRNA molecules with the right shape to target the protein, EZH2. Four computer programs listed 29 possibilities in total, but only two of these appeared in all four readouts. Of these, only miRNA-101 reduced the amount of EZH2 when precursors of this RNA were added to cancer cells.

The researchers also found that one or both of two genes that encode miRNA-101 had been lost in two-thirds of spreading prostate cancers, unleashing overexpression of EZH2.

\section{PHYSICAL CHEMISTRY}

\section{Electrons with a twist}

Phys. Rev. Lett. 101, 178301 (2008)

The direction of an electron's spin is sufficient to determine the decomposition rate of the two mirror-image forms of a molecule.

Richard Rosenberg of Argonne National Laboratory in Illinois and his colleagues drew this conclusion after firing X-rays at a magnetic iron-nickel alloy coated with thin layers of 2-butanol. This freed electrons from the alloy, and their spins aligned with its magnetic field.

With the alloy magnetized in one direction, the electrons tended to increase the rate that right-handed 2-butanol fell apart in response to light by about $10 \%$; the opposite field caused a similar effect in left-handed molecules. The two forms of the molecule are shown below.



\section{ATMOSPHERIC SCIENCES}

\section{Methane mutterings}

Geophys. Res. Lett. doi: 10.1029/2008GL036037 (2008)

After almost a decade of stability, the amount of methane in the atmosphere has been growing since the start of 2007, according to measurements taken by two global monitoring networks, AGAGE and CSIRO.

The findings are cause for concern because methane is many times more powerful than carbon dioxide as a greenhouse gas, and has so far accounted for about a fifth of the human contribution to climate change. Matthew Rigby of the Massachusetts Institute of Technology in Cambridge and their co-workers write that, by late 2007, the proportion of methane in the atmosphere was rising by 10 parts per billion per year at all monitoring stations around the world.

High bacterial methane emissions from wetlands in an unusually warm Siberia seem to have played some part in the northern hemisphere increase. The authors also suspect that an unproven drop in hydroxyl free radicals could be driving the trend.

\section{JOURNAL CLUB}

\author{
William B. McKinnon \\ Washington University, St Louis, \\ Missouri
}

\section{A planetary scientist has big hopes for a little world.}

Right now, the most exciting object in the Solar System is Saturn's diminutive moon Enceladus. Its deformed south polar region emits copious amounts of heat along the length of several young, active ridges and fractures, as well as plumes of tiny ice particles, water vapour and other chemicals.

The Cassini spacecraft equipped with plume-gas and particle analysers and clever imaging gadgetry - is currently in the neighbourhood. Seizing this opportunity, Gabriel Tobie of the University of Nantes in France and his colleagues have incorporated some of its recent measurements into theoretical models of tidal heat production on Enceladus (G. Tobie et al. Icarus 196, 642-652; 2008). Only the ebb and flow of tides could properly account for such prodigious geological activity on an icy moon that measures just 500 kilometres in diameter.

The authors start with the generally accepted idea that Enceladus has differentiated into a rock core and an icy mantle. They then show that the size of the tidal motion of the mantle is inadequate to generate the observed thermal emission, so there must be a fluid ocean sandwiched between the two solid layers. This is no great surprise, but Tobie et al. go further, showing that even if the mantle is made soft and deformable over the southern polar region (as the ice would be if it were relatively warm), a sandwiched, liquid ocean must reach at least as far as around the entire southern hemisphere.

The team imagines that, below Enceladus's south pole, tidal heating concentrates in warm, upwelling, convectively mobile ice. This, in turn, causes the cold, brittle surface layer to rupture - and the exposed warm ice sublimates, releasing trapped gases. It is a compelling picture, and one that promises to help unlock the internal activity of other icy satellites.

Discuss this paper at http://blogs. nature.com/nature/journalclub 